\title{
Role of laparoscopy during surgery at the porta hepatis
}

\author{
A Yamataka, ${ }^{1} \mathrm{MD}, \mathrm{PhD}$; G J Lane, ${ }^{1} \mathrm{MBBS}, \mathrm{PhD} ; \mathbf{H}$ Koga $,{ }^{1} \mathrm{MD}, \mathrm{PhD} ; \mathbf{J}$ Cazares, ${ }^{2} \mathrm{MD} ; \mathbf{H}$ Nakamura, ${ }^{1} \mathrm{MD}, \mathrm{PhD}$ \\ ${ }^{1}$ Department of Pediatric Surgery, Juntendo University School of Medicine, Tokyo, Japan \\ ${ }^{2}$ Department of Pediatric Surgery, Hospital Regional de Alta Especialidad Materno Infantil, Monterrey, Mexico
}

Corresponding author: A Yamataka (yama@juntendo.ac.jp)

Minimally invasive surgery in children has evolved to the extent that complex procedures can be performed with safety, with comparable outcomes to open surgery and with the advantages of minimal scarring and less pain. In this article, we describe the latest laparoscopic techniques used at Juntendo University Hospital in Japan, for treating conditions affecting the porta hepatis, focusing on biliary atresia and choledochal cysts. We also summarise our postoperative management protocols and discuss preliminary outcomes.

S Afr Med J 2014;104(11):820-824. DOI:10.7196/SAMJ.8921

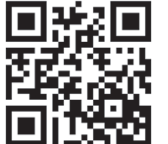

A combination of innovative technology and growing patient awareness has meant that minimally invasive surgery (MIS) for hepatobiliary disorders must be offered as a treatment option, limited only by availability and the preference and confidence of surgeons. The laparoscopic procedures performed routinely at Juntendo University Hospital in Tokyo, Japan, to treat conditions affecting the porta hepatis are presented. Both biliary atresia (BA) and choledochal cysts (CCs) involve surgical intervention centred on the porta hepatis, requiring surgeons to have a mastery of complex skills and exhaustive knowledge of anatomical variations, while parents of paediatric patients expect advantages of MIS such as improved cosmesis, less requirement for analgesia, and a reduced incidence of postoperative morbidity such as bowel adhesions and incisional hernias.

\section{Biliary atresia}

We began using laparoscopy to perform Kasai portoenterostomy (lapK) clinically in 2009, encouraged by improvements in technology, patient awareness of MIS and successful trials. ${ }^{[1]}$ Since then, several other centres in Japan have started performing lapK with inconsistent results. We attribute our results to strict adoption of the surgical principles of Kasai's original procedure..$^{[2,3]}$

\section{Choledochal cyst}

Laparoscopic excision with Roux-en-Y hepaticojejunostomy $(\mathrm{HJ})$ is well accepted for the treatment of CCs in children, in contrast to lapK. Good outcomes have been achieved in general. Here, we focus on the special features of our laparoscopic cyst excision (lapCE). We strongly believe that biliary debris/stones and protein plugs (PP) in the common channel and dilated

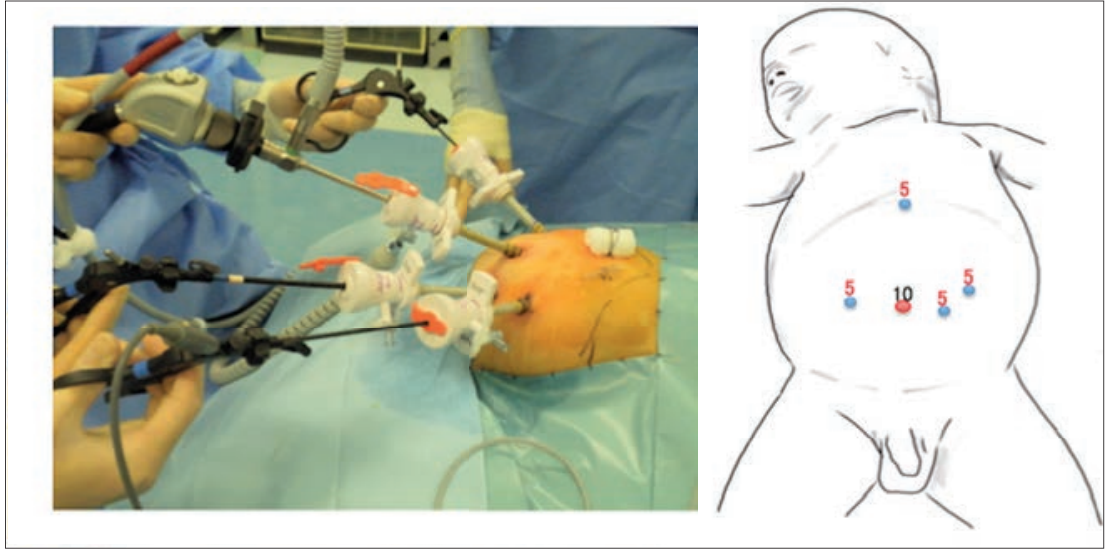

Fig. 1. Trocar positions during laparoscopic portoenterostomy. Note the $5 \mathrm{~mm}$ trocar in the epigastrium, specifically for the Ligasure device. Numbers indicate trocar size ( $\mathrm{mm}$ ).

intrahepatic bile ducts (IHBD) are the cause of postoperative cholangitis, pancreatitis and postoperative stone formation in mid- to long-term follow-up. We perform intraoperative endoscopy (IE) routinely in all our CC patients, both open and lapCE, to examine for such debris and $\mathrm{PP}$, and remove them by irrigation, if present. ${ }^{[4]}$

\section{Surgical techniques \\ Biliary atresia}

Patient/port positioning and initial preparation

Kasai portoenterostomy is performed under general anaesthesia with the patient positioned at the foot of the operating table, the surgeon at the patient's feet, an assistant with a laparoscope on the surgeon's left and another assistant on the surgeon's right. A supraumbilical $10 \mathrm{~mm}$ trocar is inserted using Hasson's open technique for a $30^{\circ}$ $5 \mathrm{~mm}$ or $10 \mathrm{~mm}$ laparoscope. Three more trocars are then inserted under laparoscopic control; one $5 \mathrm{~mm}$ trocar in each upper quadrant and one $5 \mathrm{~mm}$ trocar to the left of the umbilicus (para-umbilical port) (Fig. 1).

\section{Dissection of biliary remnants and the} porta hepatis

Adequate exposure of the porta hepatis is crucial, and is achieved by elevating the liver using a percutaneous stay suture, introduced just below the xiphoid process, to snare the round and falciform ligaments and retract the liver. Two additional percutaneous stay sutures are placed in the parenchyma of both the right and left lobes to elevate the liver for further exposure of the porta hepatis. The use of a Nathanson retractor (Teleflex Medical, UK), placed through the epigastrium, is also effective for exposing the porta hepatis for anastomosis. The cystic duct and the mid-to-distal biliary remnant are dissected using a combination of hook diathermy, a Ligasure device (Valley Lab, USA) and tissue forceps. The fibrotic biliary remnant is then transected distally at the superior border of the duodenum. The distal end of the biliary remnant is elevated to enable the right and left hepatic arteries, and the bifurcation of the main portal vein, to be visualised clearly. When dissecting the fibrotic biliary remnant, special attention must be given to the small vertical branches of the portal veins located around the 
biliary remnant at 3, 6, and 9 oclock; these drain into the caudate lobe. We are unique in that we do not use high-power hook diathermy to divide these branches because we believe diathermy causes extensive lateral thermal injury that could extend as far as the fibrotic biliary cone and damage any viable microscopic-sized bile ducts that may be present. Instead, we use a Ligasure device because it generates far less lateral thermal energy, and to prevent any risk of complications secondary to direct pressure and heat on the right or left portal veins, such as portal vein thrombosis. The Ligasure device is used through the existing para-umbilical port, or an extra $5 \mathrm{~mm}$ trocar inserted into the epigastrium to ensure that only the tips of the device make contact with the tissue to be sealed (portal vein branches); the device can be inserted into the abdomen almost vertically. When other ports are used, more than the tip of the Ligasure device may contact surrounding tissues and cause complications. The extra trocar in the epigastrium (Fig. 1, right side) is only inserted when required if the angle of the Ligasure device is not ideal (Fig. 2).

The level of transection follows Kasai's original description strictly, leaving the liver parenchyma intact (Fig. 3A). ${ }^{[2,3]}$

\section{Extracorporeal transumbilical jejunal} Roux-en-Y

The ligament of Treitz is identified and $10-15 \mathrm{~cm}$ of the jejunum distal to the ligament is exteriorised through the umbilical port site to create an extracorporeal Rouxen-Y jejunal loop. Pneumoperitoneum is terminated and we customise the Roux-en-Y limb to fit naturally into the splenic flexure after anastomosis by placing the jejunal loop at the umbilicus and bringing the distal end of the limb up to the xiphoid process. The jejunojejunostomy is performed extracorporeally. The customised Roux-en-Y limb is approximated to the native jejunum for $8 \mathrm{~cm}$ cranially to streamline flow into the distal jejunum, eliminate reflux into the Roux-en-Y limb and prevent stasis in the Roux-en-Y limb (Fig. 4). We create a $10 \mathrm{~mm}$ long antimesenteric enterotomy very near the closed end of the Roux-en-Y limb with a scalpel, unlike the vast majority who use high-voltage endoscopic diathermy, because we want to prevent lateral thermal injury to the bowel that could be the cause of leakage and scarring at the anastomosis. We then gently approximate the edges of the enterotomy temporarily with two $7 / 0$ polydioxanone sutures (PDS) (Ethicon Inc., USA) to prevent spillage of bowel contents and cut the sutures at the time of portoenterostomy. The jejunum is then returned to the abdominal cavity.

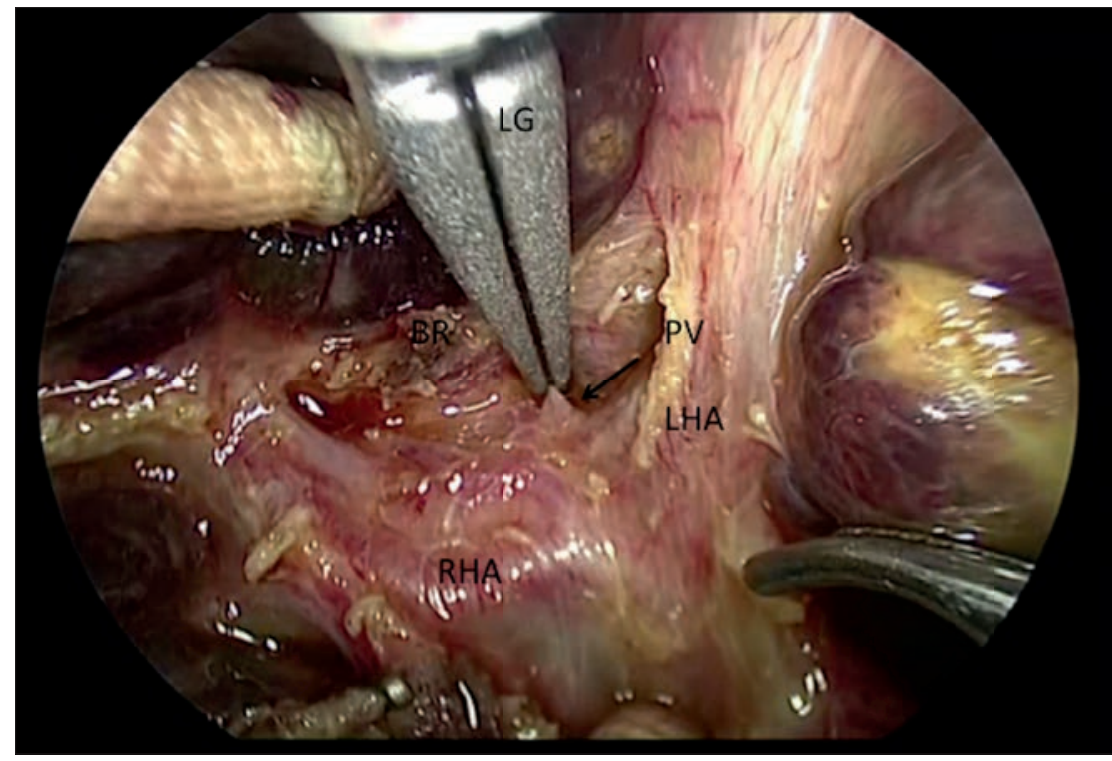

Fig. 2. An LG device being used to divide a PV branch (arrow) at the porta hepatis. (LG = Ligasure; $P V=$ portal vein; $B R=$ biliary remnant RHA = right hepatic artery; $L H A=$ left hepatic artery.)

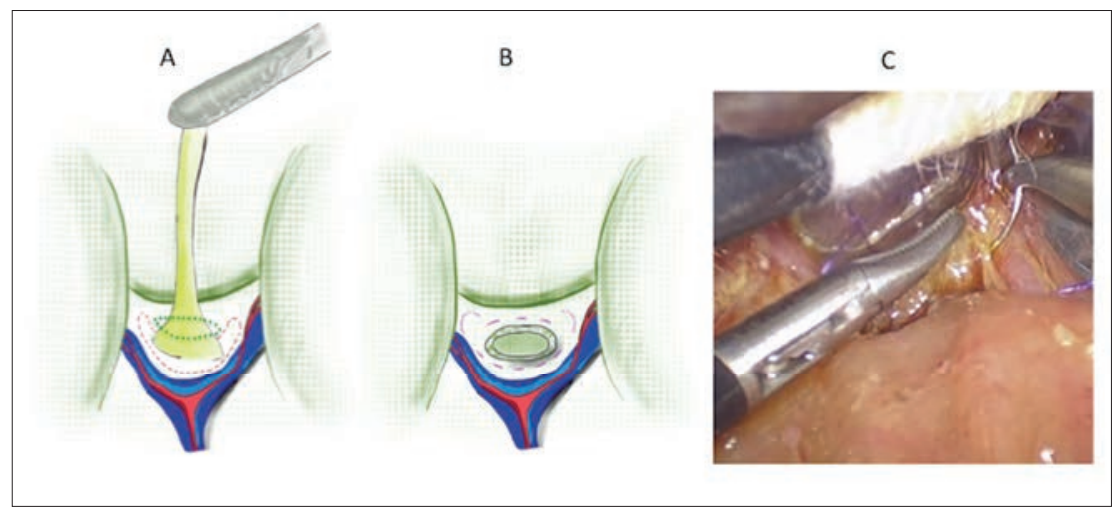

Fig. 3. Biliary remnant transection level and suturing during laparoscopic portoenterostomy. (A) Shallow transection of the biliary remnant similar to Kasai's original procedure (dotted line) compared with deep transection (broken lines). (B) Anastomotic sutures are placed between the enterotomy and liver parenchyma around the margin of the transected portal plate (broken lines). (C) Microbile duct injury is minimised by suturing only connective tissue around the biliary remnant at the 2 and 10 o'clock positions (dotted lines in B).

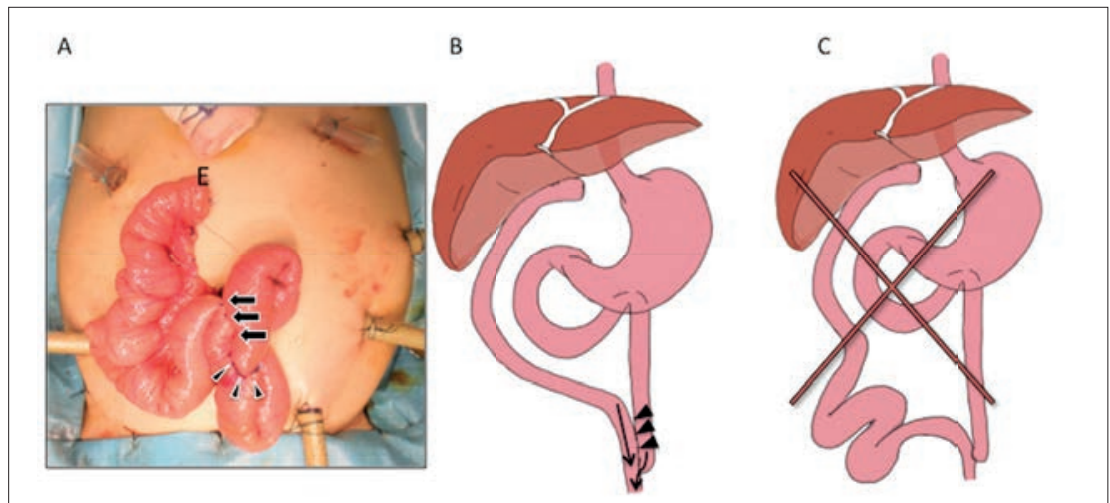

Fig. 4. Customising the Roux-en-Y limb. (A) The jejunal loop is placed at the umbilicus and the distal end (E) of the limb is brought up to the xiphoid process. (B) The customised Roux-en-Y limb is approximated to the native jejunum for $8 \mathrm{~cm}$ cranially (triangles) to streamline flow into the distal jejunum (arrows), eliminate reflux into the Roux-en-Y limb and prevent stasis in the Roux-en-Y limb. (C) Note the long jejunal limb, which can become redundant and tortuous later in life, as the patient grows, and cause bile stasis. 
Pneumoperitoneum is re-established and the closed end of the jejunal limb is brought up via a retrocolic window to the porta hepatis.

\section{Portoenterostomy}

Anastomotic sutures (5/0 or 6/0 PDS) are placed between the enterotomy and liver parenchyma around the margin of the transected portal plate (Fig. 3, B). To minimise microbile duct injury during the anastomosis, we do not place sutures in the liver parenchyma at 2 and 10 oclock, where the right and left bile ducts should be, but suture the connective tissue around the 2 and 10 o'clock positions at the porta hepatis (Fig. 3, $\mathrm{B}$ and $\mathrm{C}$ ). Also, all sutures for the anastomosis are deliberately shallow, especially at the 2 and 10 oclock positions, but deep enough to prevent leakage. A tube drain is inserted into the foramen of Winslow. Wounds are closed conventionally with infiltration of $0.25 \%$ bupivacaine for analgesia.

Postoperative care and steroid protocol Intravenous fluids and nasogastric aspiration are continued until there is confirmed recovery of bowel motility, usually from 2 - 3 days postoperatively. Careful monitoring of blood glucose, electrolytes and clotting profiles are important in the early postoperative period. Liver biochemistry may worsen during the first week regardless of the eventual outcome, but by about the 4 th week there should be a definite fall in bilirubin and consistently pigmented stools.

We administer antibiotics, cholagogues and steroids according to standard protocols. Specifically, a decreasing dose regimen of prednisolone is administered intravenously once C-reactive protein (CRP) falls below $1.0 \mathrm{mg} / \mathrm{dL}^{\left[{ }^{[5]}\right.}$ Each dose is given for 3 days, commencing with an initial dose of $4 \mathrm{mg} /$ $\mathrm{kg} / \mathrm{day}$, then $3,2,1 \mathrm{mg} / \mathrm{kg} /$ day and finishing with $0.5 \mathrm{mg} / \mathrm{kg} /$ day. This 15 -day cycle can be repeated up to four or five times if jaundice persists (total bilirubin $>1.2 \mathrm{mg} / \mathrm{dL}$ ) and there is evidence that there is clinical benefit (i.e. lower serum bilirubin or improvement in stool colour). However, if jaundice persists without evidence of clinical benefit, then only three cycles are administered and the patient is actively considered for liver transplantation (LTx). An important aspect of this protocol is if stools begin to turn pale, the cycle is either recommenced from the beginning or the previous dose is readministered, depending on circumstances. Double agent antibiotic therapy, usually a cephalosporin and an aminoglycoside, is routine and is ceased once CRP is $<0.3 \mathrm{mg} / \mathrm{dL}$. An intravenous
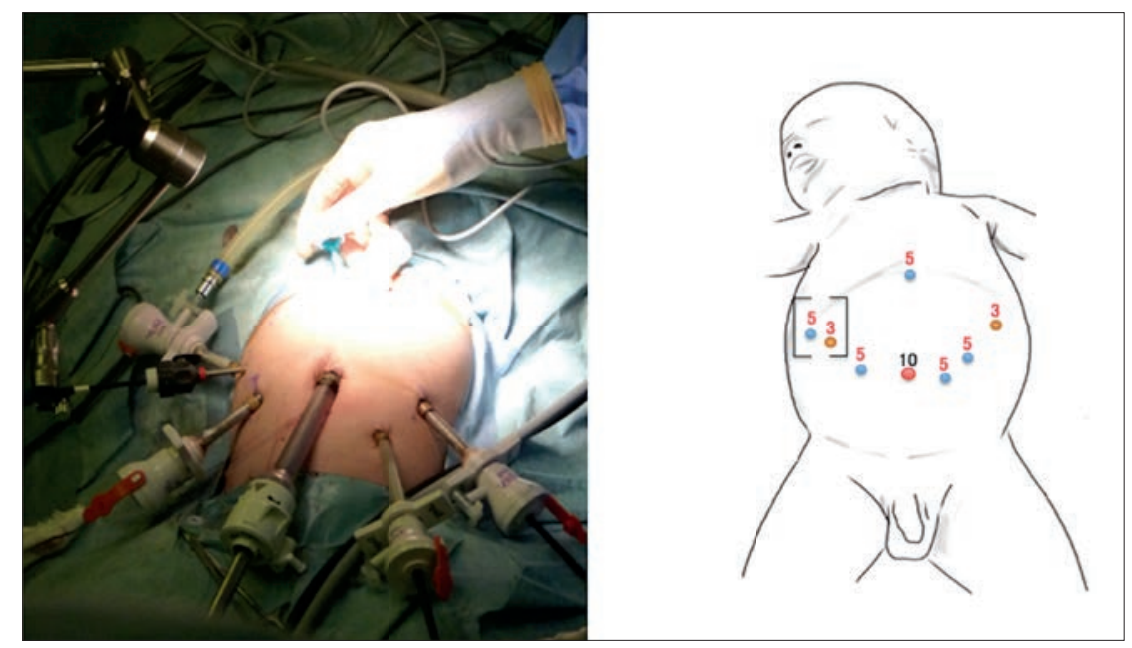

Fig. 5. Trocar positions during laparoscopic choledochal cyst excision. Note the $5 \mathrm{~mm}$ trocar in the epigastrium for the ureteroscope, and additional $3 \mathrm{~mm}$ and $5 \mathrm{~mm}$ trocars on the right for the hepaticojejunostomy. To expose the porta hepatis, a pair of Babcock forceps are inserted through the left subcostal port in the anterior axillary line (shown in the diagram, but not in the intraoperative photo). Numbers indicate trocar size $(\mathrm{mm})$.

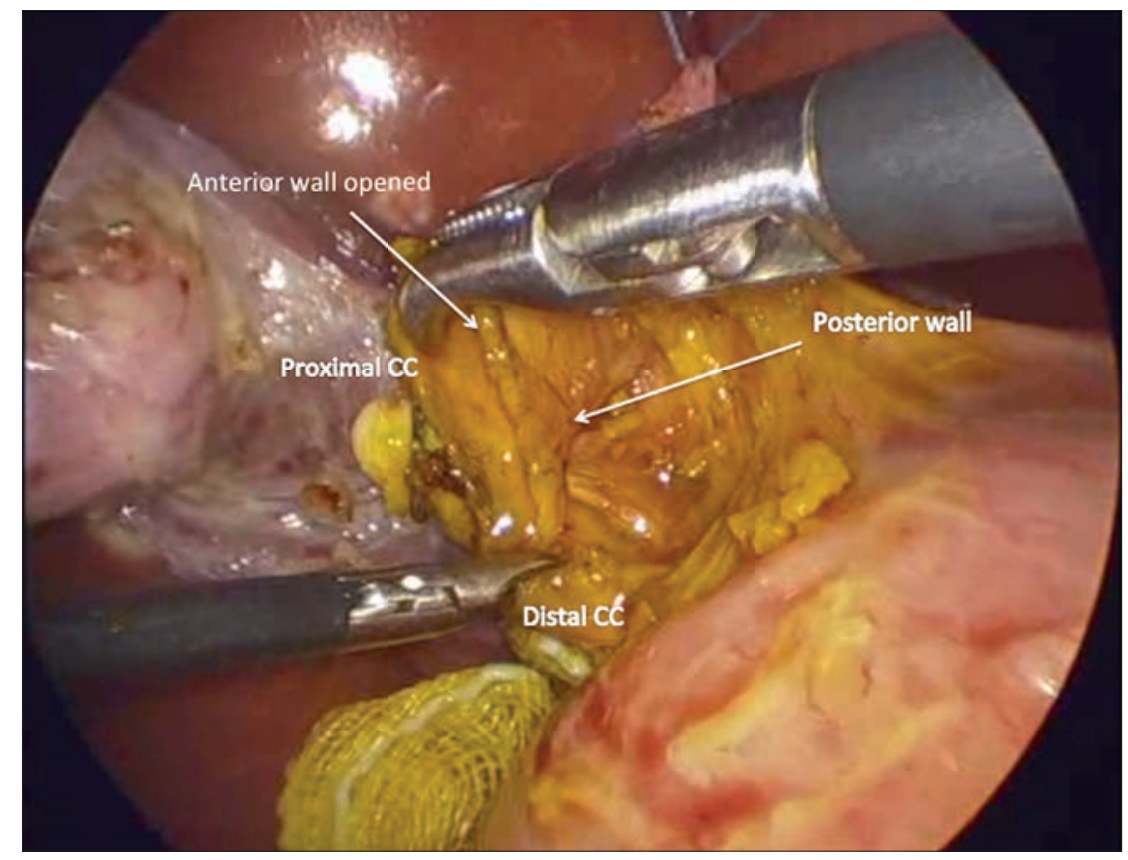

Fig. 6. Cyst dissection. The anterior wall of the cyst is incised first to allow the posterior wall to be dissected safely under direct vision. (CC = choledochal cyst.)

cholagogue (usually dehydrocholic acid) is commenced 2 days after lapK and continued until jaundice clears. Oral cholagogues such as ursodeoxycholic acid or aminoethylsulphonic acid are administered once oral feeding resumes, around 5 days after lapK, and continued thereafter. Postoperative cholangitis, defined as elevated serum bilirubin $(>2.5 \mathrm{mg} /$ $\mathrm{dL}$ ), leukocytosis and a change from normal to acholic stools in a febrile patient $\left(>38.5^{\circ} \mathrm{C}\right)$, is treated with intravenous antibiotics. Once resolved, prophylactic antibiotics such as sulfamethoxazole/trimethoprim are administered orally.

\section{Choledochal cyst}

Patient/port positioning and initial preparation

Under general anaesthesia, patients are placed in the reverse Trendelenburg position. An open Hasson technique through a supra-umbilical incision is used for placing the initial trocar for insertion of a $5 \mathrm{~mm}$ or $10 \mathrm{~mm}, 30^{\circ}$ or $45^{\circ}$ laparoscope. Carbon dioxide pneumoperitoneum is established at a pressure of $10-12 \mathrm{mmHg}$. Three additional $5 \mathrm{~mm}$ trocars are inserted in the right upper quadrant, left para-umbilical area and left upper quadrant (Fig. 5). 
Adequate exposure is achieved by elevating the liver, by introducing a percutaneous stay suture just below the xiphoid process to snare the falciform ligament and retract the liver. To expose the porta hepatis, a pair of Babcock forceps is inserted through the left subcostal port in the anterior axillary line, to grasp and elevate the gallbladder to allow the $\mathrm{CC}$ to be dissected free from surrounding structures, such as the portal vein and hepatic artery. Usually more adhesions are found between cystic CCs and the portal vein and hepatic artery than with fusiform CCs, especially in older children. In adolescents and adults, adhesions can be very dense and complicate dissection. The anterior wall of the cyst is incised first to allow the posterior wall of the cyst to be dissected safely under direct vision (Fig. 6). We believe this to be safer, especially when the cyst is inflamed and there are dense adhesions.

\section{Intraoperative cholangiography}

Intraoperative cholangiography (IC) is performed if preoperative magnetic resonance cholangiopancreatography (MRCP) is not available or fails to delineate the anatomy of the hepatopancreaticobiliary ducts, especially the anatomy of the pancreaticobiliary junction, and the presence of debris or PP in the IHBD and common channel. Usually, preoperative MRCP is accurate in the majority of cases.

\section{Intraoperative endoscopy}

For IE, an additional $5 \mathrm{~mm}$ trocar is placed in the left epigastrium for the introduction of a fine paediatric ureteroscope (Fig. 5). We use a paediatric ureteroscope specifically because it allows normal saline to flow continuously through a dedicated side channel, allowing constant visualisation and irrigation. While some surgeons suggest that laparoscopic examination is sufficient, we find that a constant flow of saline is necessary to keep the lumen of what we are examining (the common channel, pancreatic duct, IHBD, etc.) open for safe examination, and is indispensible for clearing debris and PP (Fig. 7). Without a constant flow of saline, it is very difficult to visualise anything because the lumen collapses and greatly compromises the effective examination for debris or PP; while flexible scopes have side channels, they are only designed for flushing and not for general inspection or irrigation. IE is performed in all patients unless the ureteroscope cannot be inserted smoothly into the intrapancreatic choledochus and common channel from the distal part of the CC owing to the opening being too narrow.

\section{Cyst dissection and excision}

The cystic artery is identified and divided. Dissection of the CC is initiated by removing the adjacent peritoneum using monopolar

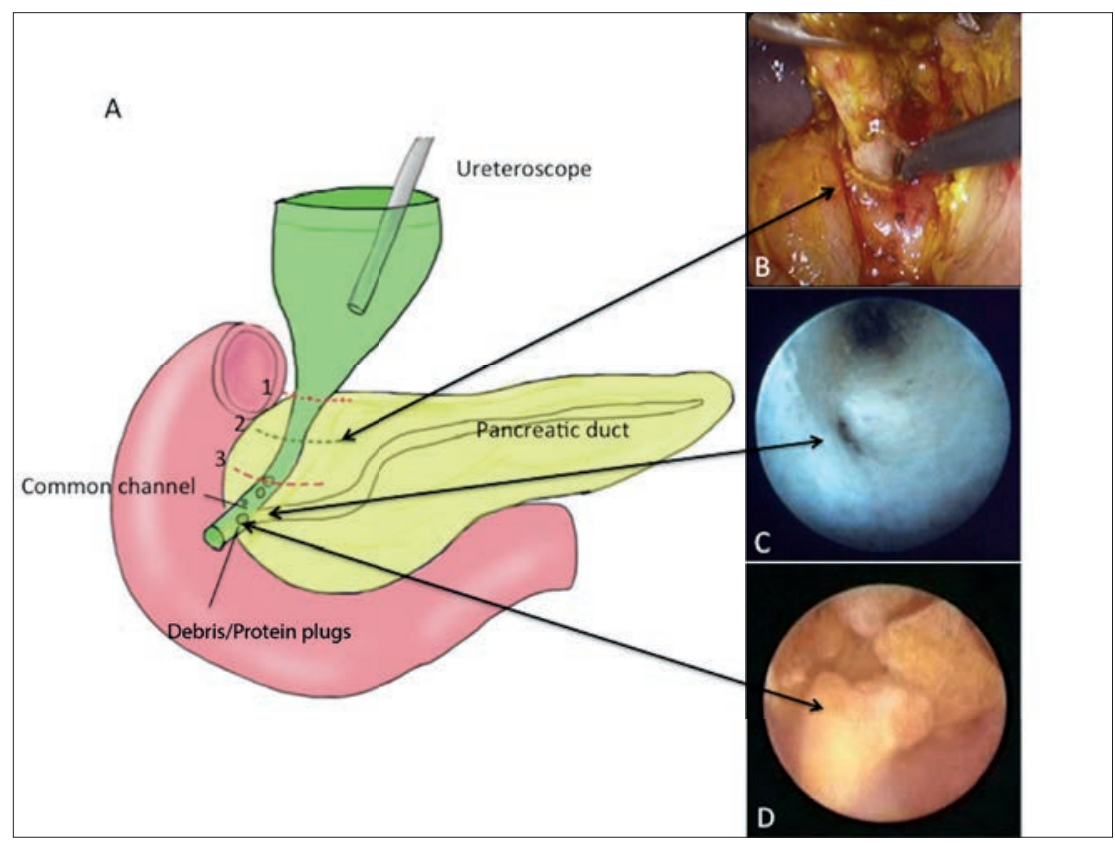

Fig. 7. Role of intraoperative endoscopy. (A) Diagram of intraoperative endoscopy. (B) Ideal level of excision is level 2. (C) The orifice of the pancreatic duct can be identified before excision. (D) Debris and protein plugs in the bile duct distal to the cyst. If excised at level 1, there is risk for residual cyst, and if excised at level 3, there is risk of pancreatic duct injury.

electrocautery and a Maryland dissector to establish a plane of dissection, beginning on the anterior wall and continuing to the medial and lateral sides, then to the distal portion. The exact level of transection of the distal common bile duct can also be determined through IE, if the orifice of the pancreatic duct in the common channel is identified (Fig. 7). After the cyst is freed, the distal part is divided as close as possible to the pancreaticobiliary junction and the stump is ligated with an endoloop. When IE cannot be performed because of a narrow opening into the intrapancreatic choledochus and common channel (common in cystic CCs), IC may be performed by placing an endoscopic metal clip at the distal end of the dissected cyst to confirm the extent of further dissection required; distally, because the clip and the confluence between the common channel, intrapancreatic choledochus and pancreatic duct can be visualised. If dissection is inadequate, the cyst can be further dissected distally and IC repeated as above until cyst dissection is adequate. The proximal cyst is excised leaving $10 \mathrm{~mm}$ of common hepatic bile duct. Finally, a cholecystectomy is performed.

\section{Extracorporeal transumbilical jejunal \\ Roux-en-Y}

The Roux-en-Y limb is constructed according to the description presented in the portoenterostomy section of BA. We wish to reiterate that we use a scalpel to create the antimesenteric enterotomy for the $\mathrm{HJ}$, not high-voltage endoscopic diathermy. The limb is customised and approximated to the native jejunum in the same way as described earlier in the BA section, and the closed end of the limb is brought up via a retrocolic window to the porta hepatis.

\section{Hepaticojejunostomy}

Our reconstructive procedure of choice is $\mathrm{HJ}$ rather than hepaticoduodenostomy (HD), which has been reported to be associated with pancreatic enzyme reflux into the biliary tree, especially when IHBD dilatation is present. $\mathrm{HD}$ is also a potential cause of mucosal damage and possible malignant change. ${ }^{[6]}$ An additional two ports $(3 / 5 \mathrm{~mm})$ are required for $\mathrm{HJ}$; one lateral right subcostal port and one between the right subcostal and right upper quadrant ports (Fig. 5). From experience, if $\mathrm{HJ}$ is performed without extra trocars, the quality of the anastomosis deteriorates, especially when the diameter of the $\mathrm{HJ}$ anastomosis is $<9 \mathrm{~mm}$. End-to-side $\mathrm{HJ}$ is performed using interrupted $5 / 0$ or $6 / 0$ absorbable sutures with the right upper 


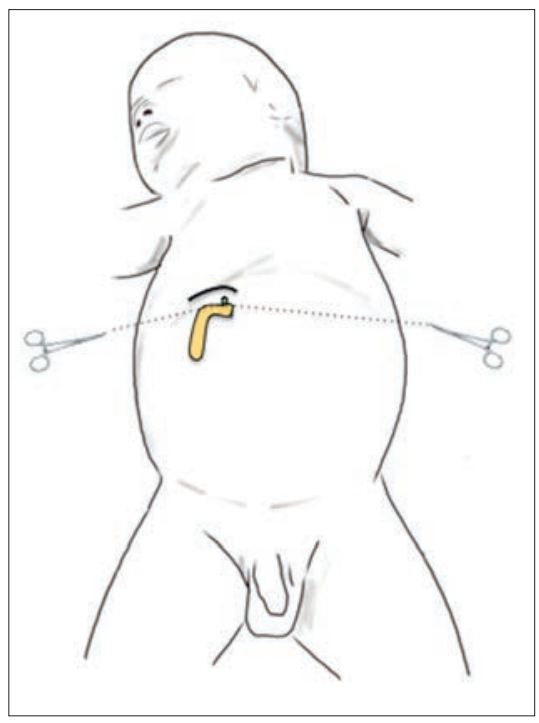

Fig. 8. Traction sutures facilitate accurate hepaticojejunostomy.

quadrant port as a needle holder in the right hand, the $5 \mathrm{~mm}$ port for the scope and the $3 \mathrm{~mm}$ subcostal port as a needle receiver in the left hand. Both the right and left edge sutures are exteriorised and used as traction sutures during anastomosis of the anterior wall to facilitate accuracy (Fig. 8), especially when the $\mathrm{HJ}$ anastomosis diameter is $<9 \mathrm{~mm}$. A tube drain is inserted in the foramen of Winslow. The resected cyst and gallbladder are extracted through the umbilicus wound. The trocars are removed and the wounds are closed.

\section{Outcomes and discussion Biliary atresia}

We are currently reviewing 15 of our lapK cases, focusing on jaundice clearance (JC) (total bilirubin $\leq 1.2 \mathrm{mg} / \mathrm{dL}$ ) and rates of survival with the native liver (SNL) as indicators of success. Of these, 13 had lapK at Juntendo University Hospital in Tokyo, Japan, and two had lapK at Hospital Regional de Alta Especialidad Materno Infantil in Monterrey, Mexico. The specialist treating surgeon at Hospital Regional was trained specifically at Juntendo University Hospital to perform lapK using identical techniques and identical postoperative protocols. JC was $93.3 \%$ (14/15 cases), took a mean of 58.5 days and required a mean of $82.0 \mathrm{mg} /$ $\mathrm{kg}$ of steroids. Postoperative cholangitis complicated $7 / 15$ cases $(46.7 \%)$. Three cases required LTx, resulting in an SNL ratio of $12 / 15(80 \%)$. LTx was indicated for portal hypertension and liver cirrhosis in cases 2 and 3 and portal hypertension

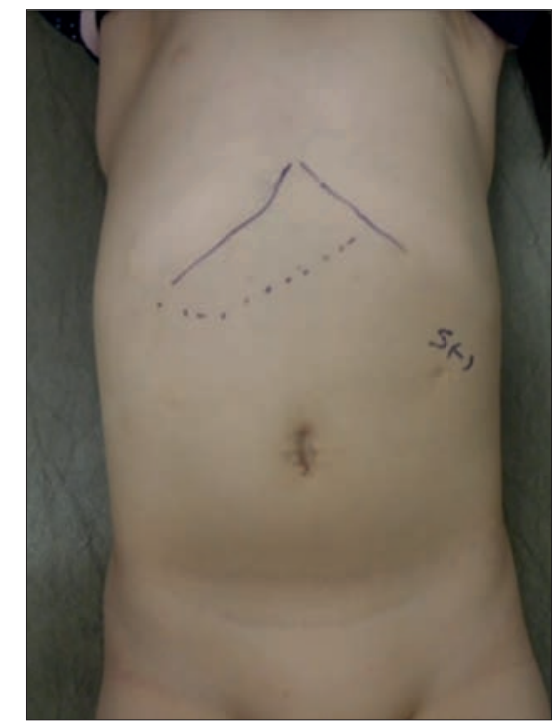

Fig. 9. Appearance of the abdomen after laparoscopic portoenterostomy. Wounds are cosmetically aesthetic. Dotted line indicates the extent of hepatomegaly. There is no splenomegaly (S-).

in case 8, 1 892, 326 and 112 days after lapK, respectively. Total bilirubin at LTx was $1.91 \mathrm{mg} / \mathrm{dL}$ in case 2 and $1.6 \mathrm{mg} / \mathrm{dL}$ in case 3; case 8 was jaundice free. In these LTx cases, intra-abdominal adhesions were much milder than in LTx performed following open Kasai, thus shortening operating times.

All SNL cases are currently jaundice free with cosmetically aesthetic wounds (Fig. 9); however, one case, a 5-year-old boy, is currently having percutaneous transhepatic cholangiodrainage for a bile leak at the porta hepatis.

To date, we have been able to perform lapK safely in all cases, with high SNL to JC ratios, even though the operative time for lapK is long (8 hours 50 minutes). We expect operating time to shorten with experience gained from performing more cases.

\section{Choledochal cyst}

We had 28 CC patients who underwent lapCE between 2009 and 2014. Two cases required conversion (one case of open laparotomy and one case of minilaparotomy) and were omitted, leaving 26 cases; 19 females and 7 males. Mean (range) age at lapCE was $5.0(1.0-14.2)$ years, and mean body weight was $16.5(8.0-47.0) \mathrm{kg}$. CCs were fusiform in 19 cases and cystic in 7 cases. Five patients had IHBD dilatation. Of the 19 fusiform CC cases, 16 had HJ diameters of $6-9 \mathrm{~mm}$, while the remaining 3 fusiform CC cases and 7 cystic-type CC cases had $\mathrm{HJ}$ diameters $>10 \mathrm{~mm}$. IE of both the common channel and IHBD were performed in 16 cases (all fusiform); the remaining 10 had only IE for IHBD because the ureteroscope could not be inserted into the intrapancreatic choledochus and common channel. During IE of the common channel, all 16 fusiform CC cases had PP in the common channel, which was successfully removed by irrigation with normal saline from the side channel of the ureteroscope (massive PP in 4, moderate in 10, minimal in 2). Debris was present in the IHBD in 13 of the $26 \mathrm{CC}$ cases (moderate in 6 , minimal in 7); the remaining 13 had no debris in the IHBD. There were no intraoperative complications. Estimated mean blood loss was minimal at $20 \mathrm{~mL}$. Although all patients are well after a mean follow-up of 2.8 years (range 1 month - 5.2 years) with cosmetically aesthetic wounds, there were three postoperative complications. The first case was one with massive PP who developed pancreatitis 8 months postoperatively even though all debris had been removed during IE; this was probably due to newly created fine debris $(3 \mathrm{~mm} \times 3 \mathrm{~mm})$. The pancreatitis was treated by conservative medical management and there have been no further episodes. The second case was one with cystic CCs who developed duodenal obstruction postoperatively. At exploratory laparoscopy, the third part of the duodenum was found to be compressed by the Roux-en-Y limb that had been inadequately fixed to the colonic mesentery. Once the sutures between the Roux-en-Y limb and colonic mesentery were released laparoscopically, the postoperative recovery course was uneventful. The third case had anastomotic leakage that was treated by mini-laparotomy.

Our routine lapCE involves the use of extra trocars, IE customising the length of the Roux-en-Y limb. Operating time is longer for lapCE, but postoperative pain is minimised, allowing patients to be discharged earlier.

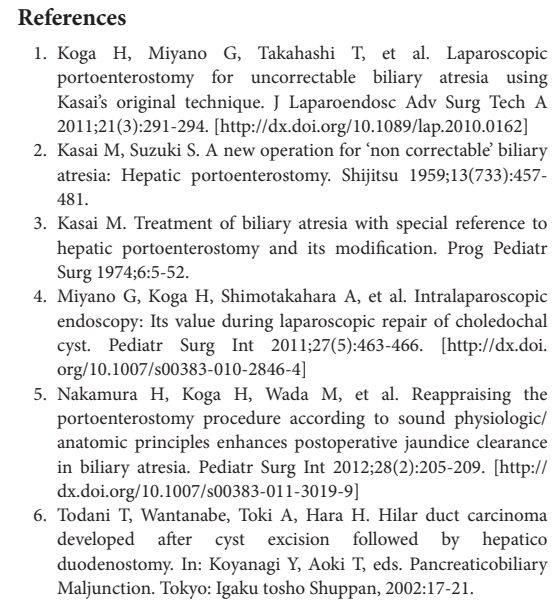
portoenterostomy for uncorrectable biliary atresia using Kasai's original technique. J Laparoendosc Adv Surg Tech A 2011;21(3):291-294. [http://dx.doi.org/10.1089/lap.2010.0162] 2. Kasai M, Suzuki S. A new operation for 'non correctable' biliary atresia: Hepatic portoenterostomy. Shijitsu 1959;13(733):457481.

3. Kasai M. Treatment of biliary atresia with special reference to hepatic portoenterostomy and its modification. Prog Pediatr Surg 1974;6:5-52.

4. Miyano G, Koga H, Shimotakahara A, et al. Intralaparoscopic endoscopy: Its value during laparoscopic repair of choledochal cyst. Pediatr Surg Int 2011;27(5):463-466. [http://dx.doi. org/10.1007/s00383-010-2846-4]

5. Nakamura H, Koga H, Wada M, et al. Reappraising the portoenterostomy procedure according to sound physiologic/ anatomic principles enhances postoperative jaundice clearance in biliary atresia. Pediatr Surg Int 2012;28(2):205-209. [http:// dx.doi.org/10.1007/s00383-011-3019-9]

6. Todani T, Wantanabe, Toki A, Hara H. Hilar duct carcinoma developed after cyst excision followed by hepatico duodenostomy. In: Koyanagi Y, Aoki T, eds. Pancreaticobiliary Maljunction. Tokyo: Igaku tosho Shuppan, 2002:17-21. 